\title{
Effect of reverse intersystem crossing rate to suppress efficiency roll-off in organic light-emitting diodes with thermally activated delayed fluorescence emitters
}

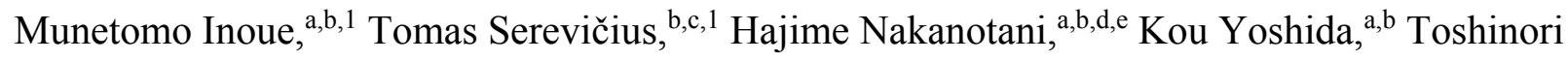
Matsushima, ${ }^{\text {,d }}$ Saulius Juršènas,${ }^{\mathrm{c}}$ Chihaya Adachi ${ }^{\mathrm{a}, \mathrm{b}, \mathrm{d}, \mathrm{e}, *}$

a Department of Applied Chemistry, 744 Motooka, Nishi, Fukuoka 819-0395, Japan

${ }^{\mathrm{b}}$ Center for Organic Photonics and Electronics Research, Kyushu University, 744 Motooka, Nishi, Fukuoka 819-0395, Japan

${ }^{\mathrm{c}}$ Institute of Applied Research, Vilnius University, Sauletekio 9-III, LT-10222 Vilnius, Lithuania

d Japan Science and Technology Agency (JST), ERATO, Adachi Molecular Exciton Engineering Project, 744 Motooka, Nishi, Fukuoka 819-0395, Japan

e International Institute for Carbon Neutral Energy Research, Kyushu University, 744 Motooka, Nishi, Fukuoka 819-0395, Japan

${ }^{1} \mathrm{M}$. Inoue and T. Serevičius contributed equally to this work.

*Corresponding author.

C. Adachi, Center for Organic Photonics and Electronics Research (OPERA), 744 Motooka, Nishi, Fukuoka 819-0395, Japan

Tel: +81-92-802-6920; E-mail: adachi@cstf.kyushu-u.ac.jp

\section{Keywords}

organic light-emitting diodes (OLEDs), thermally activated delayed fluorescence (TADF), roll-off characteristics, singlet-triplet annihilation (STA), triplet-triplet annihilation (TTA)

(C) 2015. This manuscript version is made available under the Elsevier user license http://www.elsevier.com/open-access/userlicense/1.0/ 


\begin{abstract}
Electroluminescence efficiency roll-off in organic light-emitting diodes with thermally activated delayed fluorescence emitters 1,2-bis(carbazol-9-yl)-4,5-dicyanobenzene (2CzPN) and 3-(9,9dimethylacridin-10(9H)-yl)-9H-xanthen-9-one (ACRXTN) is investigated by considering intramolecular exciton relaxation processes. Electroluminescence efficiency roll-off at high current density is dramatically suppressed using ACRXTN as an emitter instead of $2 \mathrm{CzPN}$ because of suppressed bimolecular exciton annihilation processes such as singlet-triplet and triplet-triplet annihilation. The rate constant of reverse intersystem crossing from triplet to singlet excited states of ACRXTN is about 300 times higher than that of $2 \mathrm{CzPN}$, decreasing triplet exciton density and suppressing exciton annihilation processes under optical and electrical excitation.
\end{abstract}




\section{Introduction}

Because organic light-emitting diodes (OLEDs) can exhibit nearly 100\% internal electroluminescence (EL) quantum efficiency ( $\eta_{\text {int }}$ ), they are attractive for next-generation lighting sources as well as display applications. Although nearly $100 \% \eta_{\text {int }}$ has been achieved, the decrease of EL efficiency, known as rolloff, at high brightness [1] is a serious problem limiting the development of high-brightness OLEDs. Over the past two decades, two types of organic luminescent compounds have mainly been developed as emitters for OLEDs. OLEDs using fluorescent materials as emitters exhibit rather weak roll-off characteristics, although their $\eta_{\text {int }}$ is intrinsically limited to $25 \%$ because only $25 \%$ of excitons, corresponding to singlet excitons, could be harvested under electrical excitation [1,2]. In contrast, although efficient room-temperature phosphorescent materials such as tris(2-phenylpyridinato)iridium(III) $\left(\operatorname{Ir}(\text { ppy })_{3}\right)$ can provide nearly $100 \% \eta_{\text {int, }}$, the large accumulation of triplet excitons in an emitter layer causes considerable bimolecular deactivation, i.e., triplet-triplet annihilation (TTA), because of the rather long lifetimes of triplet excitons (a few $\mu$ s) compared with those of typical singlet excitons (a few ns) [1-5]. In fact, according to reported simulations [1,4,6], the efficiency roll-off of OLEDs using (E)-2-(2-(4(dimethylamino)styryl)-6-methyl-4H-pyran-4-ylidene)malononitrile as a fluorescent emitter and $\operatorname{Ir}(\mathrm{ppy})_{3}$ as a phosphorescent emitter has been ascribed to mainly singlet-triplet annihilation (STA) $[1,6]$ and TTA $[1,4]$, respectively.

Recently, we proposed a unique triplet harvesting process in OLEDs, namely thermally activated delayed fluorescence (TADF) [7-9]. In OLEDs using TADF molecules as an emitter (TADF-OLEDs), although a maximum $\eta_{\text {int }}$ of nearly $100 \%$ has been achieved, they also exhibit substantial roll-off behavior similar to that of phosphorescence-based OLEDs [8-10]. To understand the origin of the roll-off of TADFOLEDs, here we investigate the exciton annihilation processes in OLEDs containing the sky-blue TADF molecules 1,2-bis(carbazol-9-yl)-4,5-dicyanobenzene (2CzPN) and 3-(9,9-dimethylacridin-10(9H)-yl)9H-xanthen-9-one (ACRXTN), and reveal that both STA and TTA contribute to the roll-off of TADF- 
OLEDs. We demonstrate that the rate constant of reverse intersystem crossing (RISC) from triplet to singlet excited states $\left(k_{\mathrm{RISC}}\right)$ of ACRXTN $\left(k_{\mathrm{RISC}}=\sim 10^{5} \mathrm{~s}^{-1}\right)$ is about 300 times higher than that of $2 \mathrm{CzPN}$ $\left(k_{\mathrm{RISC}}=\sim 10^{3} \mathrm{~s}^{-1}\right)$, and this larger $k_{\mathrm{RISC}}$ of ACRXTN decreases the triplet exciton density, suppressing STA and TTA under electrical excitation.

\section{Experimental}

The molecular structures of TADF emitters 2CzPN and ACRXTN are shown in Figure 1(a). OLEDs containing $2 \mathrm{CzPN}$ and ACRXTN as emitters were fabricated by conventional vacuum deposition on glass substrates coated with a 100-nm-thick indium tin oxide (ITO) layer under a base pressure of around $7 \times$ $10^{-4} \mathrm{~Pa}$. The OLEDs possessed the structure glass substrate / ITO $(100 \mathrm{~nm}) / \alpha-\mathrm{NPD}(30 \mathrm{~nm}) / \mathrm{mCP}(10$ nm) / 5 mol\% 2CzPN- or ACRXTN-doped mCP (15 nm) / PPT (10 nm) / TPBi (40 nm) / LiF $(0.8 \mathrm{~nm}) /$ Al (100 nm), where $\alpha$-NPD, mCP, PPT and TPBi are 4,4'-bis[ $N$-(1-naphthyl)- $N$-phenyl-amino]biphenyl, $N, N^{\prime}$-dicarbazolyl-3,5-benzene, 2,8-bis(diphenylphosphoryl)dibenzo-[b,d]thiophene, and 2,2',2”-(1,3,5benzenetriyl)tris[1-phenyl-1H-benzimidazole], respectively. Current density $(J)$-voltage $(V)$-external quantum efficiency $\left(\eta_{\mathrm{EQE}}\right)$ characteristics and EL spectra of the OLEDs were measured using an integrating sphere connected to a source meter (2400, Keithley Instruments) and a photonic multichannel analyzer (PMA-12, Hamamatsu Photonics). For optical measurements, 100-nm-thick mCP films doped with $5 \mathrm{~mol} \% 2 \mathrm{CzPN}$ or ACRXTN were fabricated on quartz substrates by vacuum deposition. Photoluminescence (PL) spectra and PL quantum yields (PLQYs) of these films were measured by a spectrofluorometer (FluoroMax-4, Horiba Jobin Yvon) and PLQY measurement system (C9920-02, Hamamatsu Photonics), respectively. PL lifetimes of the films were measured under vacuum using a streak camera (C4334, Hamamatsu Photonics), and a nitrogen gas laser (MNL200, Lasertechnik) with an excitation wavelength of $337 \mathrm{~nm}$ and pulse width of $500 \mathrm{ps}$ as an excitation source. The rate constants 
shown in Figure 1(b) were calculated from the values of PLQY and PL lifetime as previously reported [10]. The transient EL response of the OLEDs during long pulse voltage excitation was also measured using a photomultiplier tube (C9525-02, Hamamatsu Photonics) and pulse generator (WF 1974, NF corporation). The pulse width was $500 \mu \mathrm{s}$ and the repetition frequency was $0.5 \mathrm{~Hz}$ for this measurement.

\section{Results and discussion}

PL and EL spectra, and $J-V$ and $\eta_{\mathrm{EQE}}-J$ characteristics of the OLEDs with $2 \mathrm{CzPN}$ and ACRXTN are presented in Figure 2 . The maximum $\eta_{\mathrm{EQE}}\left(\eta_{\mathrm{EQE}, \max }\right)$, current density when $\eta_{\mathrm{EQE}}$ decreases to half of the initial $\eta_{\mathrm{EQE}}\left(J_{0}\right), \eta_{\mathrm{EQE}}$ at $1,000 \mathrm{~cd} \mathrm{~m}^{-2}$, rate constant of STA $\left(k_{\mathrm{STA}}\right)$, and rate constant of TTA $\left(k_{\mathrm{TTA}}\right)$ are summarized in Table 1. These OLEDs showed almost the same sky-blue EL spectra [Figure 2(a)]. The slight red shift of EL spectra compared with the PL spectra of the composite films was ascribed to an optical microcavity effect. Before the onset of the $\eta_{\mathrm{EQE}}$ roll-off, the $\eta_{\mathrm{EQE}}$, max values of the OLEDs with $2 \mathrm{CzPN}$ and ACRXTN were similar values of $10.3 \%$ and $12.1 \%$, respectively. The small difference in $\eta_{\mathrm{EQE}}$ max originates from the different PLQYs of $2 \mathrm{CzPN}(81 \%)$ and ACRXTN (92\%). Because both OLEDs showed similar $J-V$ characteristics [Figure 2(b)], it is expected that the carrier balance factor would have a small influence on $\eta_{\mathrm{EQE}}$ max. However, $J_{0}$ of the OLEDs with ACRXTN and $2 \mathrm{CzPN}$ differed markedly. At $L=1,000 \mathrm{~cd} \mathrm{~m}^{-2}, \eta_{\mathrm{EQE}}$ of the OLED with ACRXTN $\left(\eta_{\mathrm{EQE}}=11.0 \%\right)$ was about five times larger than that of the OLED with $2 \mathrm{CzPN}\left(\eta_{\mathrm{EQE}}=2.2 \%\right)$, indicating that the roll-off at high current density was strongly suppressed using ACRXTN. Here, $k_{\mathrm{STA}}$ and $k_{\mathrm{TTA}}$ were estimated using rate equations based on STA and TTA models, [10]

$$
\begin{gathered}
\frac{d N_{\mathrm{S}}}{d t}=-\left(k_{\mathrm{r}}^{\mathrm{S}}+k_{\mathrm{ISC}}\right) N_{\mathrm{S}}+k_{\mathrm{RISC}} N_{\mathrm{T}}-k_{\mathrm{STA}} N_{\mathrm{S}} N_{\mathrm{T}}+\alpha k_{\mathrm{TTA}} N_{\mathrm{T}}^{2}+\frac{J}{4 d e} \\
\text { and } \frac{d N_{\mathrm{T}}}{d t}=k_{\mathrm{ISC}} N_{\mathrm{S}}-\left(k_{\mathrm{RISC}}+k_{\mathrm{nr}}^{\mathrm{T}}\right) N_{\mathrm{T}}-(1+\alpha) k_{\mathrm{TTA}} N_{\mathrm{T}}^{2}+\frac{3 J}{4 d e},
\end{gathered}
$$


where $N_{\mathrm{S}}$ and $N_{\mathrm{T}}$ are the singlet and triplet exciton densities, respectively, $k_{\mathrm{r}}^{\mathrm{S}}$ is the rate constant of radiative emission from the lowest singlet excited state $\left(\mathrm{S}_{1}\right), k_{\mathrm{ISC}}$ is the rate constant of intersystem crossing (ISC) from $\mathrm{S}_{1}$ to the lowest triplet excited state $\left(\mathrm{T}_{1}\right), k_{\mathrm{nr}}{ }^{\mathrm{T}}$ is the rate constant of non-radiative decay from $\mathrm{T}_{1}$ to the ground state, and $\alpha$ is the singlet exciton production ratio via TTA, which is 0.25 according to spin statistics [10]. Fitting the experimental $\eta_{\mathrm{EQE}}-J$ data with eqs. (1) and (2) [Figure 2(c)] yields $k_{\mathrm{STA}}=$ $2.1 \times 10^{-11} \mathrm{~cm}^{3} \mathrm{~s}^{-1}$ and $k_{\mathrm{TTA}}=5.0 \times 10^{-15} \mathrm{~cm}^{3} \mathrm{~s}^{-1}$ for $2 \mathrm{CzPN}$, and $k_{\mathrm{STA}}=1.9 \times 10^{-11} \mathrm{~cm}^{3} \mathrm{~s}^{-1}$ and $k_{\mathrm{TTA}}=$ $5.1 \times 10^{-15} \mathrm{~cm}^{3} \mathrm{~s}^{-1}$ for ACRXTN (Table 1). Although $k_{\mathrm{STA}}$ and $k_{\mathrm{TTA}}$ of the OLEDs with ACRXTN and $2 \mathrm{CzPN}$ are quite similar, a large difference in their roll-off characteristics was observed. Actually, $N_{\mathrm{T}}$ in the OLEDs with ACRXTN, calculated using eqs. (1) and (2), is much smaller than that in the OLEDs with $2 \mathrm{CzPN}$ at all current densities [Figure 2(c)]. The experimental $J, \eta_{\mathrm{EQE}}$, and $\eta_{\mathrm{EQE}} / \eta_{\mathrm{EQE}}$ max for the OLEDs containing ACRXTN and $2 \mathrm{CzPN}$ at different $N_{\mathrm{T}}$ are summarized in Table 2 . It is interesting that the $\eta_{\mathrm{EQE}} / \eta_{\mathrm{EQE}}$, max values for $2 \mathrm{CzPN}$ are the same as those for $\mathrm{ACRXTN}$ at the same $N_{\mathrm{T}}$ while there is no clear relationship between other parameters, indicating that the roll-off characteristics are essentially controlled by $N_{\mathrm{T}}$.

To investigate the origin of the suppressed roll-off behavior and lower $N_{\mathrm{T}}$ for ACRXTN than $2 \mathrm{CzPN}$, the intramolecular exciton relaxation processes of $\mathrm{ACRXTN}$ and $2 \mathrm{CzPN}$ were examined. The rate constants of the intramolecular relaxation processes were calculated from the PLQYs and PL lifetimes of the composite films according to ref. 10 . Table 3 summarizes the calculated $\lambda_{\max }, \Delta E_{\mathrm{ST}}, \Phi_{\text {total }}$, $\Phi_{\text {prompt}}, \Phi_{\text {delayed, }}, \tau_{\text {prompt }}, \tau_{\text {delayed }}, k_{\mathrm{r}}^{\mathrm{S}}, k_{\mathrm{ISC}}, k_{\mathrm{RISC}}$, and $k_{\mathrm{nr}}{ }^{\mathrm{T}}$, for ACRXTN and $2 \mathrm{CzPN}$, where $\lambda_{\max }$ is the PL peak wavelength; $\Delta E_{\mathrm{ST}}$ is the energy gap between $\mathrm{S}_{1}$ and $\mathrm{T}_{1} ; \Phi_{\text {total }}, \Phi_{\text {prompt, }}$, and $\Phi_{\text {delayed }}$ are the PLQYs of the total, prompt, and delayed components, respectively; and $\tau_{\text {prompt }}$ and $\tau_{\text {delayed }}$ are the PL lifetimes of the prompt and delayed components, respectively. Although there is no large difference in $k_{\mathrm{r}}^{\mathrm{S}}$ and $k_{\mathrm{ISC}}$ between $2 \mathrm{CzPN}$ and ACRXTN, it is noteworthy that $k_{\mathrm{RISC}}$ of ACRXTN is about 300 times higher than that of 
$2 \mathrm{CzPN}$ because of its smaller $\Delta E_{\mathrm{ST}}$ of $33 \mathrm{meV}$. The larger $k_{\mathrm{RISC}}$ makes it possible to decrease the $N_{\mathrm{T}}$ under electrical excitation, resulting in the suppression of STA and TTA.

To further investigate how $k_{\mathrm{RISC}}$ affects the STA and TTA characteristics of emitters at high current densities, the roll-off characteristics when the OLEDs were driven under pulse voltage excitation were measured (Figure 3). We assume that the OLED breakdown observed at high current densities and voltages is caused by melting of organic layers due to Joule heating. The Joule heating-induced breakdown $\underline{\text { is suppressed by a reduction of device active areas and use of high-thermal-conductivity substrates and }}$ short pulse voltages because of suppressed Joule heating [11,12]. Therefore, a very high voltage of up to $\underline{48 \mathrm{~V} \text { could be applied to our OLEDs under the pulse voltage excitation, enabling very high current }}$ injection of up to $4.5 \mathrm{~A} \mathrm{~cm}^{-2}$ without irreversible device breakdown. The $J-V$ characteristics of both the OLEDs measured under the pulse voltage excitation agree well each other (see Figure $\mathrm{S} 1$ in the Supplementary Information). The $\eta_{\mathrm{EQE}}-J$ curves measured under pulse voltage excitation exhibited rolloff characteristics similar to those measured under DC conditions below $100 \mathrm{~mA} \mathrm{~cm}{ }^{-2}$ [Figure 3(a) and (b)]. At high current density (over $100 \mathrm{~mA} \mathrm{~cm}^{-2}$ ), the roll-off was still greater for the 2CzPN-based OLEDs than the ACRXTN-based ones. Here, we calculated $N_{\mathrm{RISC}}\left(=k_{\mathrm{RISC}} \times N_{\mathrm{T}}\right), N_{\mathrm{STA}}\left(=k_{\mathrm{STA}} \times N_{\mathrm{S}} \times N_{\mathrm{T}}\right)$, and $N_{\mathrm{TTA}}\left[=(1+\alpha) \times k_{\mathrm{TTA}} \times N_{\mathrm{T}}^{2}\right]$ using eqs. (1) and (2), which are plotted as a function of current density in Figure 3(a) and (b). NRISC is the number per unit time of the decrease of triplet excitons or increase of singlet excitons by RISC. NSTA is the number per unit time of the decrease of singlet excitons by STA, while $N_{\mathrm{TTA}}$ is the number per unit time of the decrease of triplet excitons by TTA. $N_{\mathrm{STA}}$ and $N_{\mathrm{TTA}}$ were higher than $N_{\text {RISC }}$ at most current densities for the OLEDs with $2 \mathrm{CzPN}$, resulting in the observed marked efficiency roll-off. Conversely, at most current densities, NRISC was higher than $N_{\text {STA and }} N_{\text {TTA in the }}$ OLEDs with ACRXTN and $N_{\text {STA }}$ and $N_{\text {TTA }}$ in the OLEDs with ACRXTN were lower than those of the OLEDs with 2CzPN, effectively suppressing the efficiency roll-off in ACRXTN based OLEDs. These 
results suggest that RISC, STA, and TTA compete during operation of the OLEDs, and that the faster RISC overcomes STA and TTA in the OLEDs with ACRXTN.

Additionally, an initial change in EL intensity during pulse voltage excitation at a high current density of $4.5 \mathrm{~A} \mathrm{~cm}^{-2}$ was observed between the OLEDs with 2CzPN and ACRXTN [Figure 3(c)]. The EL intensity suddenly decreased after an initial increase in EL intensity for the 2CzPN-based OLEDs while the ACRXTN-based OLEDs showed no such EL decrease. Similar decreases in EL and PL intensities have been observed for fluorescent OLEDs and composite films of TADF materials and attributed to STA $[6,13,14]$. We found that the PL intensities of $2 \mathrm{CzPN}$ composite films decreased under optical excitation at high light intensities of over $40 \mathrm{~W} \mathrm{~cm}^{-2}$, although decreases of PL intensities were not observed at lower excitation intensities for the $2 \mathrm{CzPN}$ composite films or at all for the ACRXTN composite films (see Figure S2 in the Supplementary Information). Therefore, we conclude that the initial decrease of the EL and PL intensities observed for $2 \mathrm{CzPN}$ is further proof of the occurrence of strong STA. There is a possibility that triplet-polaron annihilation (TPA) also causes the efficiency roll-off. However, the contribution of TPA is quite small because of the small TPA rate constant $k_{\text {TPA }}$ of $<1.0 \times$ $10^{-12} \mathrm{~cm}^{-3} \mathrm{~s}^{-1}[6,10,15]$

\section{Conclusions}

We investigated the roll-off characteristics of TADF-OLEDs with 2CzPN and ACRXTN as emitters. We revealed that efficient RISC is important to suppress roll-off in TADF-OLEDs. In fact, the large $k_{R I S C}$ of $3.2 \times 10^{5} \mathrm{~s}^{-1}$ of ACRXTN dramatically suppressed both STA and TTA processes. We conclude that molecular design of TADF materials with small $\Delta E_{\mathrm{ST}}$ is requisite for not only obtaining high $\eta_{\mathrm{EQE}}$ but also suppressing efficiency roll-off. 


\section{Acknowledgements}

This work was supported by the Japan Science and Technology Agency (JST), ERATO, Adachi Molecular Exciton Engineering Project, and a Grant-in-aid and Fellowship from the Japan Society for the Promotion of Science (JSPS). T. Serevičius acknowledges support of the project "Promotion of Student Scientific Activities" (VP1-3.1-ŠMM-01-V-02-003) from the Research Council of Lithuania.

\section{References}

[1] C. Murawski, K. Leo, M. C. Gather, Adv. Mater. 25 (2013) 6801.

[2] N. C. Giebink, S. R. Forrest, Phys. Rev. B 77 (2008) 235215.

[3] M. A. Baldo, D. F. O’Brien, Y. You, A. Shoustikov, S. Sibley, M. E. Thompson, S. R. Forrest, Nature 395 (1998) 151.

[4] M. A. Baldo, C. Adachi, S. R. Forrest, Phys. Rev. B 62 (2000) 10967.

[5] C. Adachi, M. A. Baldo, M. E. Thompson, S. R. Forrest, J. Appl. Phys. 90 (2001) 5048.

[6] Y. Zhang, M. Whited, M. E. Thompson, S. R. Forrest, Chem. Phys. Lett. 495 (2010) 161.

[7] A. Endo, M. Ogasawara, A. Takahashi, D. Yokoyama, Y. Kato, C. Adachi, Adv. Mater. 21 (2009) 4802.

[8] S. Y. Lee, T. Yasuda, H. Nomura, C. Adachi, Appl. Phys. Lett. 101 (2012) 093306.

[9] H. Uoyama, K. Goushi, K. Shizu, H. Nomura, C. Adachi, Nature 492 (2012) 234.

[10] K. Masui, H. Nakanotani, C. Adachi, Org. Electron. 14 (2013) 2721.

[11] Y. Setoguchi, C. Adachi, J. Appl. Phys. 108 (2010) 064516.

[12] H. Yamamoto, H. Kasajima, W. Yokoyama, H. Sasabe, C. Adachi, Appl. Phys. Lett. 86 (2005) $\underline{083502 .}$

[13] T. Furukawa, H. Nakanotani, M. Inoue, C. Adachi, Sci. Rep. 5 (2015) 8429. 
[14] H. Nakanotani, T. Furukawa, C. Adachi, Adv. Opt. Mater. (2015), in press, doi.org/10.1002/adom.201500236.

[15] D. Kasemann, R. Brückner, H. Fröb, K. Leo, Phys. Rev. B 84 (2011) 115208. 
Table 1. Summary of $\eta_{\mathrm{EQE}}$ max, $J_{0}, \eta_{\mathrm{EQE}}$ at $1,000 \mathrm{~cd} \mathrm{~m}^{-2}, k_{\mathrm{STA}}$, and $k_{\mathrm{TTA}}$ of OLEDs containing $2 \mathrm{CzPN}$ and ACRXTN as emitters.

\begin{tabular}{cccccc}
$\begin{array}{c}\mathrm{TADF} \\
\text { materials }\end{array}$ & $\begin{array}{c}\eta_{\mathrm{EQE}, \max } \\
{[\%]}\end{array}$ & $\begin{array}{c}J_{0} \\
{\left[\mathrm{~mA} \mathrm{~cm}^{-2}\right]}\end{array}$ & $\begin{array}{c}\eta_{\mathrm{EQE}} \text { at } 1,000 \mathrm{cdm}^{-2} \\
{[\%]}\end{array}$ & $\begin{array}{c}k_{\mathrm{ST} A} \\
{\left[10^{-11} \mathrm{~cm}^{3} \mathrm{~s}^{-1}\right]}\end{array}$ & $\begin{array}{c}k_{\mathrm{TT} A} \\
{\left[10^{-15} \mathrm{~cm}^{3} \mathrm{~s}^{-1}\right]}\end{array}$ \\
\hline $2 \mathrm{CzPN}$ & 10.3 & 1.6 & 2.2 & 2.1 & 5.0 \\
$\mathrm{ACRXTN}$ & 12.1 & 104 & 11.0 & 1.9 & 5.1 \\
\hline
\end{tabular}

Table 2. Summary of $J, \eta_{\mathrm{EQE}}$, and $\eta_{\mathrm{EQE}} / \eta_{\mathrm{EQE} \text {, max }}$ of OLEDs containing $2 \mathrm{CzPN}$ and ACRXTN at different $N_{\mathrm{T}}$.

\begin{tabular}{ccccc} 
TADF materials & $\begin{array}{c}N_{\mathrm{T}} \\
{\left[10^{17} \mathrm{~cm}^{-3}\right]}\end{array}$ & $\begin{array}{c}J \\
{\left[\mathrm{~mA} \mathrm{~cm}^{-2}\right]}\end{array}$ & $\begin{array}{c}\eta_{\mathrm{EQE}} \\
{[\%]}\end{array}$ & $\eta_{\mathrm{EQE}} / \eta_{\mathrm{EQE}, \max }$ \\
\hline \multirow{2}{*}{$2 \mathrm{CzPN}$} & 1.0 & 0.1 & 8.7 & 0.84 \\
& 5.0 & 0.6 & 6.6 & 0.64 \\
\hline \multirow{2}{*}{ ACRXTN } & 10.0 & 1.8 & 4.8 & 0.47 \\
& 1.0 & 7.8 & 10.3 & 0.85 \\
& 5.0 & 51.0 & 7.7 & 0.64 \\
\hline
\end{tabular}


Table 3. Summary of $\lambda_{\max }, \Delta E_{\mathrm{ST}}, \Phi_{\text {total }}, \Phi_{\text {prompt, }} \Phi_{\text {delayed, }} \tau_{\text {prompt, }} \tau_{\text {delayed }}, k_{\mathrm{r}}^{\mathrm{S}}, k_{\mathrm{ISC}}, k_{\mathrm{RISC}}$, and $k_{\mathrm{nr}}^{\mathrm{T}}$ of $2 \mathrm{CzPN}$ and ACRXTN molecules.

\begin{tabular}{|c|c|c|c|c|c|c|c|c|c|c|c|}
\hline $\begin{array}{c}\text { TADF } \\
\text { materials }\end{array}$ & $\begin{array}{c}\lambda_{\max } \\
{[\mathrm{nm}]}\end{array}$ & $\begin{array}{c}\Delta E_{\mathrm{ST}} \\
{[\mathrm{meV}]}\end{array}$ & $\begin{array}{l}\Phi_{\text {total }} \\
{[\%]}\end{array}$ & $\begin{array}{c}\Phi_{\text {prompt }} \\
{[\%]}\end{array}$ & $\begin{array}{c}\Phi_{\text {delayed }} \\
{[\%]}\end{array}$ & $\begin{array}{c}\tau_{\mathrm{p}} \\
{[\mathrm{ns}]}\end{array}$ & $\begin{array}{c}\tau_{\mathrm{d}} \\
{[\mathrm{ms}]}\end{array}$ & $\begin{array}{c}k_{\mathrm{r}}^{\mathrm{S}} \\
{\left[10^{7} \mathrm{~s}^{-1}\right.}\end{array}$ & $\begin{array}{r}k_{\mathrm{ISC}} \\
{\left[10^{7} \mathrm{~s}\right.}\end{array}$ & $\begin{array}{l}k_{\mathrm{RISC}} \\
{\left[10^{5} \mathrm{~s}^{-1}\right.}\end{array}$ & $\begin{array}{c}k_{\mathrm{nr}}^{\mathrm{T}} \\
{\left[10^{5} \mathrm{~s}^{-1}\right]}\end{array}$ \\
\hline $2 \mathrm{CzPN}$ & 471 & 90 & 81 & 66 & 15 & 19 & 164 & 3.5 & 1.8 & 0.011 & 0.0097 \\
\hline ACRXTN & 478 & 33 & 92 & 74 & 18 & 26 & 2.4 & 2.2 & 0.79 & 3.2 & 1.0 \\
\hline
\end{tabular}

(a)

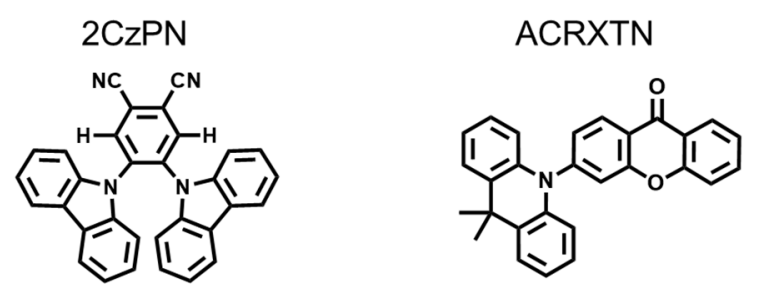

(b)

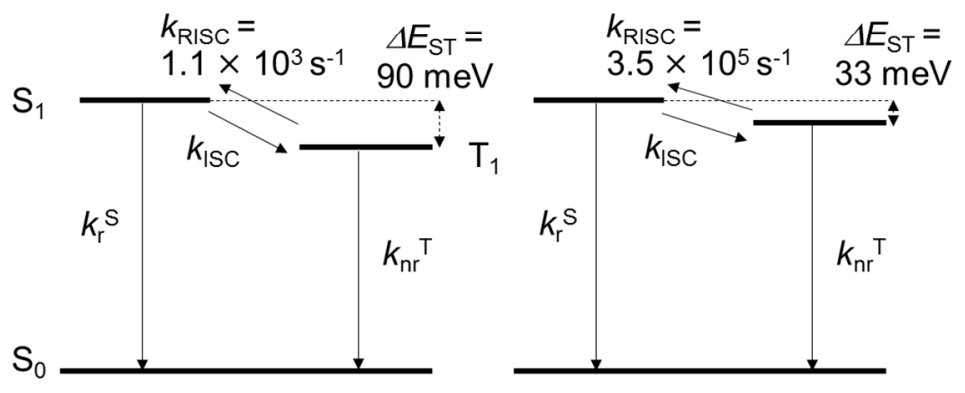

Figure 1. (a) Molecular structures of $2 \mathrm{CzPN}$ and $\mathrm{ACRXTN}$ and (b) illustration of intramolecular relaxation processes of TADF materials. The rate constants of each intramolecular relaxation process are summarized in Table 3. 
(a)

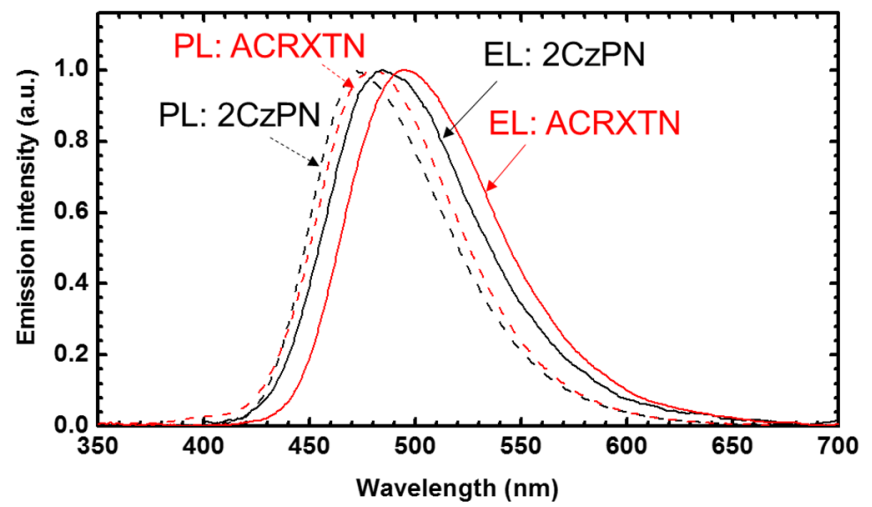

(b)

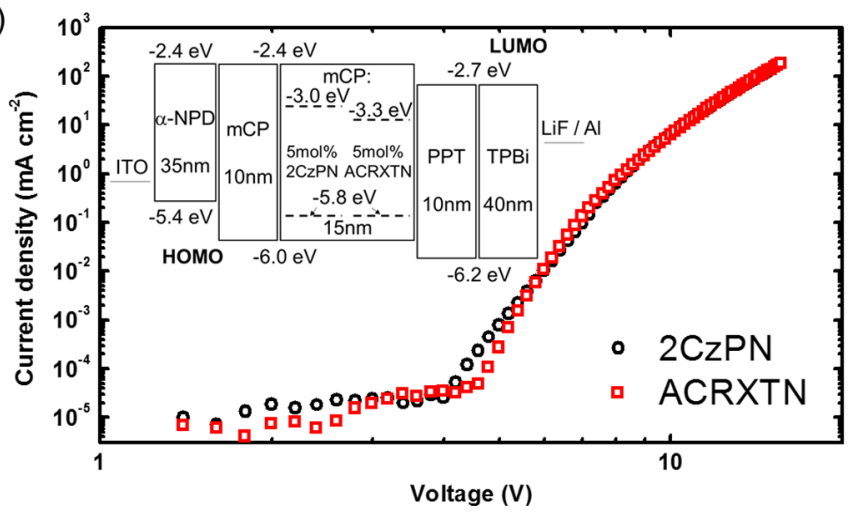

(c)

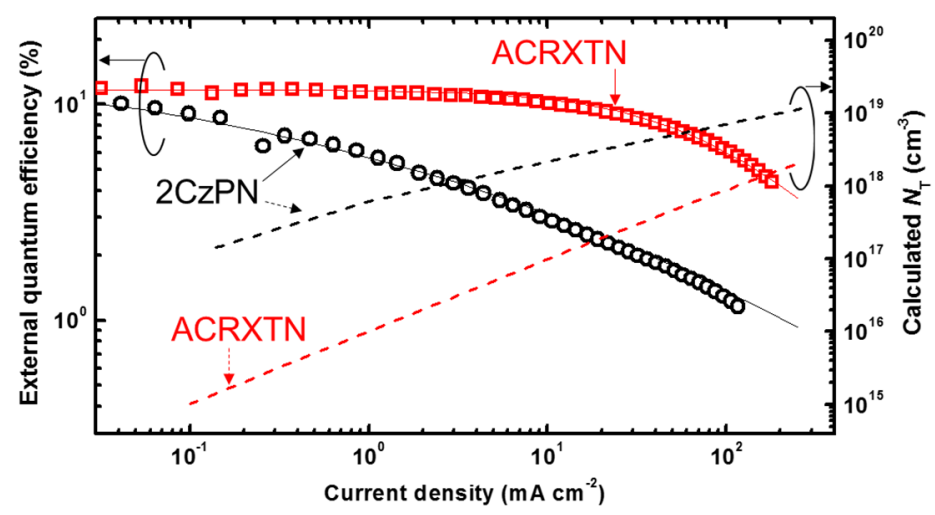

Figure 2. (a) EL spectra of OLEDs containing $2 \mathrm{CzPN}$ and ACRXTN as emitters measured at $50 \mathrm{~mA} \mathrm{~cm}^{-}$ 2 and PL spectra of composite films, and (b) $J-V$ and (c) $\eta_{\mathrm{EQE}}-J$ characteristics of the same OLEDs. The inset of (b) is an energy-level diagram of the OLEDs containing $2 \mathrm{CzPN}$ and ACRXTN as emitters. The solid and dashed lines in (c) are the fitting results and calculated $N_{\mathrm{T}}$ using the rate equations (1) and (2) based on STA and TTA models [ref. 10]. It was confirmed that shapes of EL spectra are independent of applied current densities. 
(a)

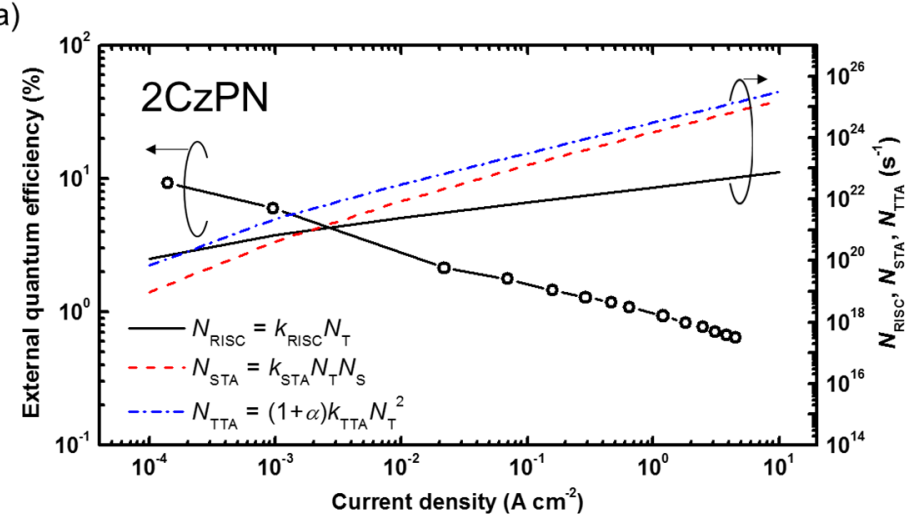

(b)

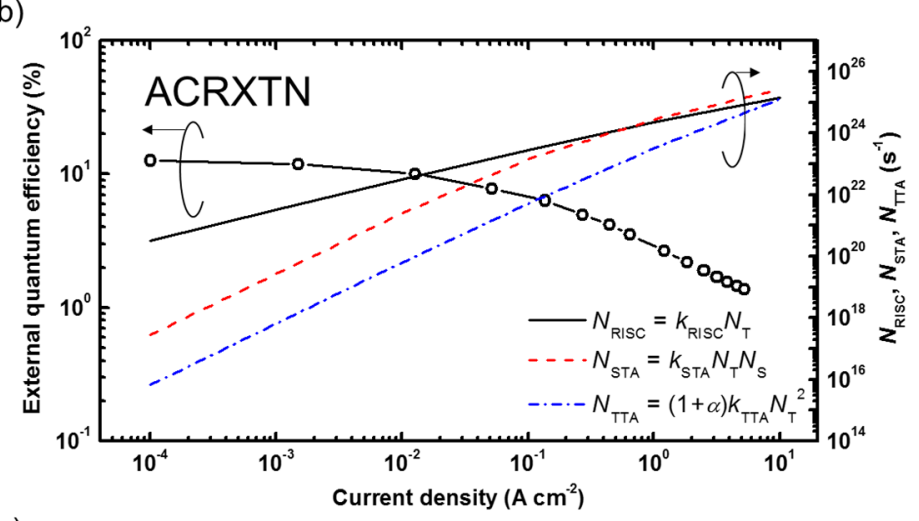

(c)

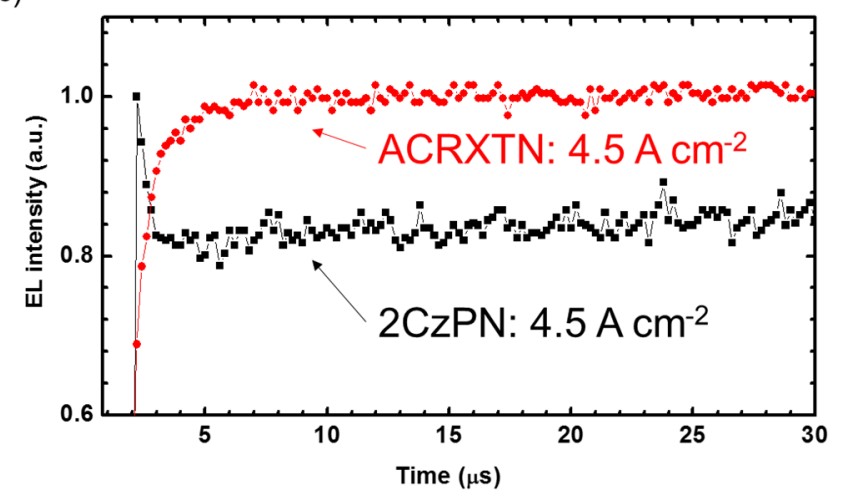

Figure 3. $\eta_{\mathrm{EQE}}-J$ characteristics of OLEDs containing (a) $2 \mathrm{CzPN}$ and (b) ACRXTN driven by pulsed voltages with a pulse width of $500 \mu$ s and repetition frequency of $0.5 \mathrm{~Hz}$. (c) Change of EL intensity of OLEDs containing 2CzPN and ACRXTN under pulse voltage excitation at a high current density of 4.5 $\mathrm{A} \mathrm{cm}{ }^{-2}$. The solid, dashed, and dot-dashed lines in (a) and (b) are $N_{\mathrm{RISC}}\left(=k_{\mathrm{RISC}} \times N_{\mathrm{T}}\right), N_{\mathrm{STA}}\left(=k_{\mathrm{STA}} \times N_{\mathrm{S}}\right.$ $\left.\times N_{\mathrm{T}}\right)$, and $N_{\mathrm{TTA}}\left[=(1+\alpha) \times k \mathrm{TTA} \times N_{\mathrm{T}}^{2}\right]$, respectively, calculated using rate equations (1) and (2). 


\section{Graphical abstract}
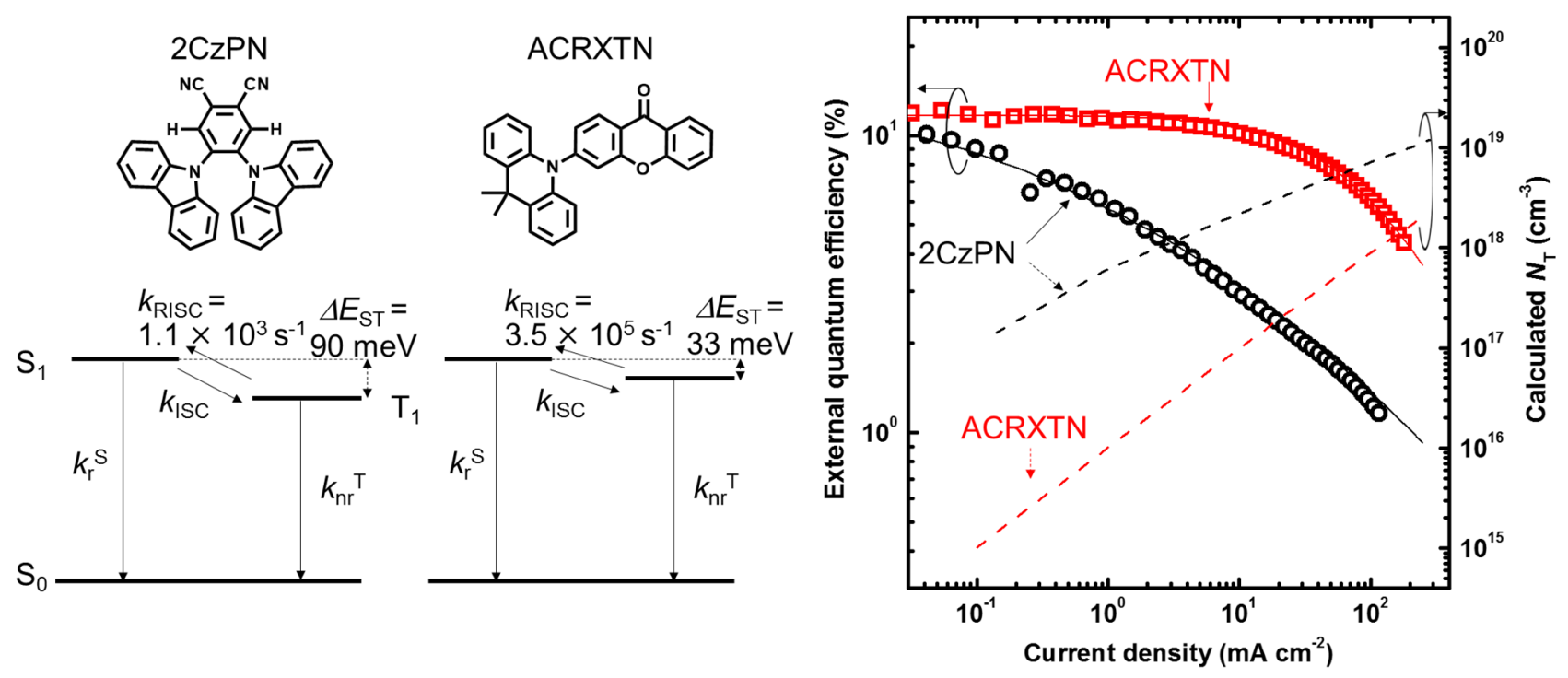